\title{
Nasal Adhesions - A New Perspective
}

\author{
Babar Rafiq Khan, Muhammad Saleem, Khizer Saleem, Jamil Ahmed, Lyla Shahbaz
}

\begin{abstract}
Objective: The objective of the study is to explore various causes of adhesions or faults what different surgeons commonly do and how to combat this problem. Study design: Randomized Control Trial. Settings: ENT Unit-I, Allied Hospital, Faisalabad. Duration: July, 2016 to June, 2018. Methodology: 500 patients undergoing septoplasty and SMR, over a period of 2 years were included in this study. Those requiring combination procedures were excluded. The study involved 400 males and 100 females. The age range was 15-75 years with a mean age of 34 years. All patients had a pre-operative nasal packing by undertraining medical officers using a roll gauze soaked in 4\% Xylocaine with Topical Adrenaline $1: 1000$ in a ratio of 50:50 half an hour before surgery. 300 cases were operated under local anesthesia with no intravenous supplement and the remaining 200 under General Anesthesia. Results: 20 patients (04\%) presented with adhesions out of which 15 were operated under General Anesthesia and 5 under local anesthesia. All these patients belonged to the non-splinted group. 07 patients (1.4\%) complained of slight discomfort due to splints particularly those made of the cut drips. 03 patients $(0.6 \%)$ complained of the unsightly look of the anterior ends of the splints as they were secured with stitches over the columella. Crust formation was the main problem encountered during the splinting period. Conclusion: Splints have been found to be very effective in preventing adhesion formation which are sometimes very troublesome. However, because of the very low incidence, (04\%) in our studies compared to (8.1\%) in other series after a single procedure on the nose, one must be very cautious in placing splints and should be placed where the surgeon strongly fears the formation of adhesions, such as in difficult cases. Suggestions: Prevention from unwanted adhesions is the best strategy to avoid adhesions.
\end{abstract}

Keywords: Septoplasty, SMR, Endoscopic, Splints.

Corresponding Author

Submitted for Publication: 14-12-2018

Accepted for Publication: 01-05-2019

DR. BABAR RAFIQ KHAN, Associate Professor, ENT-I, Faisalabad Medical University, Allied Hospital, Faisalabad-Pakistan

Contact / Email: +92 300-9650856, hina4babar@yahoo.com

Citation: Khan BR, Saleem M, Saleem K, Ahmed J, Shahbaz L. Nasal Adhesions - A New Perspective. APMC 2019;13(2):147-9.

\section{INTRODUCTION}

Adhesions or scar tissue (synaechia) is a common occurrence following any nasal surgery adhesions develop when two opposing raw mucosal surfaces remain in contract for some time in the post-operative period. Intranasal splints have been used to prevent intranasal adhesions between the nasal septum and lateral nasal wall and to support the septal position ${ }^{1}$. After turbinectomy the use of intranasal packing is important, Velasco et al. ${ }^{2}$ observed that the nasal bleeding in bilateral partial inferior turbinectomy was less in cases of nasal pack as compared to cases in which nasal packing was not used. The key point is the proper positioning of the splint on the septum.

Adhesions Molecules and Cellular Recruitment: Cellular trafficking is integral to human immune response because it allows cells to be selectively recruited from the bloodstream into sites of tissue information. Cellular recruitment into sites of allergic reactions is an example of such trafficking.

Numerous inflammatory cells are present in the nasal mucosa and in nasal secretions of allergic patients, during allergen exposure but not in healthy subjects. The molecules responsible for adhesion on leukocytes belong to different families. Such as integrin family, which can combine non-covalently to form LFA1, Mac-I and p150, 95 respectively ${ }^{3}$. Another set of adhesion proteins are VLA antigen family. L-Selection ${ }^{4}$ adhesion molecules on the vascular endothelial cell surface include ICAM-I, ICAM-2, E-Selectin, P-Selectin and VCAM-I.
Leucocytes may also be activated directly by their interaction with adhesion molecules on activated endothelial cells.

Exposure of Endothelial cells to IL-I or TNF induces endothelial expression of ICAM-I, E-Selectin \& VCAM-I and enhances adhesion of Eosinophils, Basophils and Neutrophils 5 .

Consistent with these observations were the findings that Neutrophils did not express VLA-4, whereas Eosinophils and Basophils did. After adhering to vascular endothelial cells, Eosinophils leave the circulation and enter local inflammatory sites by migrating across the Endothelium.

\section{METHODOLOGY}

Study Design: Randomized Control Trial.

Settings: ENT Unit-I, Allied Hospital, Faisalabad-Pakistan.

Duration: July, 2016 to June, 2018.

Sample Technique: Non probability sampling technique.

Methods: 500 patients undergoing septoplasty and SMR, over a period of 2 years were included in this study. Those requiring combination procedures were excluded. The study involved 400 males and 100 females. The age range was $15-75$ years with a mean age of 34 years. All patients had a pre-operative nasal packing by undertraining medical officers using a roll gauze soaked in 4\% Xylocaine with Topical Adrenaline 1:1000 in a ratio of 50:50 half an hour before surgery. 300 cases were operated under local anesthesia with no intravenous supplement and the remaining 200 under General Anesthesia. 
Splinting was done only in 10 patients using cut pieces of drips in appropriate sizes and silicon rubber preformed splints. The remaining 490 patients were simply packed with liquid paraffin soaked rolled gauze for 48 hours. All splints were removed after a mean of 8.5 days (range 7-10 days). All patients were seen daily and suction clearance carried out. Patients were examined in OPD and the more was checked for adhesions on the 15th day of the removal of splints. Statistical analysis was done using $\mathrm{CHI}$ Square and students ' $\mathrm{t}$ ' test.

\section{RESULTS}

20 patients (04\%) presented with adhesions out of which 15 were operated under General Anesthesia and 5 under local anesthesia. All these patients belonged to the non-splinted group. 07 patients (1.4\%) complained of slight discomfort due to splints particularly those made of the cut drips.

03 patients $(0.6 \%)$ complained of the unsightly look of the anterior ends of the splints as they were secured with stitches over the columella. Crust formation was the main problem encountered during the splinting period.

\section{DISCUSSION}

It is surprising that despite being one of the most common nasal procedures, the literature gives very little attention to adhesions formation after surgery.

Predisposing Factors for The Formation of Adhesions

A. Extensive surgery with tearing of the mucous membrane.

B. Unnecessary trauma to the mucous membrane during operation by instruments, suction, improper use of microdebrider, $, 6,7,8$ or aggressive use of laser resulting in ulceration of the mucous membrane.

C. Inability to remove crusts, blood clots, thick secretions in the postoperative period, i.e. inadequate postoperative care.

D. Patient neglects postoperative attendance.

E. High deviated septum not corrected at the time of operation. This results in unnecessary mucosal trauma to middle turbinate and the lateral wall of the nose as it restricts the access to the ostiomeatal complex 9,10 during surgery and in postoperative period. This mucosal trauma to the two opposing surfaces lead to formation of adhesions.

Prevention of Post-Operative Adhesions: The endoscopic surgeon should develop skilled and meticulous operative technique and take diligent care not to damage unnecessarily the mucous membrane during the operation especially while dealing Frontal Sinus for chronic rhinosinusitis with nasal polyps ${ }^{11,12,13}$. Skull Base Surgery and surgery for various sinuses should later on be confirmed by imaging of paranasal sinuses for any remnants ${ }^{14}$. Clinically seen that the Lamina Push Test is an alternative to the Globe Push Test for identifying the medial orbit during endoscopic sinus surgery ${ }^{15}$. Intraoperative navigation is an important tool for the treatment of isolated blowout fractures of the medial orbital wall. Enhanced Irrigant Delivery System can be delivered to the ethmoid sinuses directly following Ethmoid Punch Sinusotomy ${ }^{16}$. If meticulous preoperative preparation is done, bleeding should be minimal and unnecessary trauma with suction and other instruments is avoided. Nasal packing when performed at the end of the operation, adds to the problem of mucosal trauma especially to the opposing raw surfaces and contributes to the postoperative adhesions. Surgeons insert various types of nasal packing either Merocal, fingerstalls packed with ribbon gauze or various types of splints available commercially in the ethmoid cavity. Author's preferred technique is not to use any packing at the end of the operation unless the bleeding is significant, in which case Kelsostat ${ }^{\mathrm{TM}}$ (calcium alginate) pack is inserted and removed after 24 hours. A small anterior wedge resection of the middle turbinate has been found very successful in keeping the middle turbinate away from the lateral wall of the nose. It also facilitates excellent approach to the ethmoid sinuses for a postoperative cavity care, thus preventing adhesions. If there is a significant trauma to the mucous membrane, then appropriate postoperative regime should be instituted to prevent formation of adhesions. This involves following measures to be taken by the patient and the surgeon.

By the Patient: It is author's routine practice to hand over written instructions to the patients when they are discharged following endoscopic sinus surgery.

Patients are instructed to use alkaline nasal douche several times a day. This involves taking a small jug of lukewarm water, which is boiled and cooled, to which two teaspoonfuls of sodabicarb (baking soda) is added. This solution is then taken into 20-ml. syringe and the nasal cavity is syringed with the head down position in front of a wash basin. The alkaline solution is a mucolytic one, which dissolves the excessive mucus secretions, that occurs in the postoperative period. The manual cleansing also removes blood clots and crusts which form in ethmoid cavity in the postoperative period. Patients are advised to perform nasal douche for at least four to six weeks following the operation. By this time the ethmoid cavity is gradually epithelialized. Patients are also given steroid nasal spray for four to six weeks. Patients are instructed to attend the postoperative follow up examination regularly.

By the Surgeon: The surgeon should take diligent care of the ethmoid cavity in the postoperative period. This involves the following:

a. Use of local anesthetic such as four percent cocaine with 1.1000 adrenaline, or four percent lignocaine on a piece of Merocel or a cotton wool pledget to anaesthetize the nose may be necessary in some cases.

b. With 0-degree endoscope and sterile suction remove mucus, blood clots and crusts from the nasal and ethmoid cavity. In doing so, divide small fibrinous bands that form between the two adjoining surfaces. Antibiotic and steroid cream may be applied to the raw mucous membrane.

It is extremely important that the surgeon should not use excessive force in removing the crusts and the blood clots in the early postoperative period. If undue force is used, it will not only be painful for the patient but will result in removing the thin delicate epithelium that forms under these crusts. Once the new epithelium develops in the Ethmoid cavity, these crusts will become loose, and then can be gently removed under direct vision with the use of endoscope. Depending upon the extent of 
surgery and the original disease, the Ethmoid cavity will heal over a period of several weeks, until then the patient should be followed up in the clinic at varying intervals which should be "tailor made". Diligent cavity care will only minimize postoperative adhesions. Inspite of meticulous cavity care some adhesions may form in the ethmoid cavity, however, these are minor and asymptomatic and do not require any surgical treatment.

\section{CONCLUSION}

Splints have been found to be very effective in preventing adhesion formation which are sometimes very troublesome. However, because of the very low incidence, $(04 \%)$ in our studies compared to $(8.1 \%)$ in other series after a single procedure on the nose, one must be very cautious in placing splints and should be placed where the surgeon strongly fears the formation of adhesions, such as in difficult cases.

\section{REFERENCES}

1. Tang S, Kacker A. Should intransal splint be used after nasal septal surgery? Laryngoscope. 2012;122(8):1647-8.

2. Velasco LC, Arima LM, Tiago RS. Assessment of symptoms improvement following nasal septoplasty with or without turbinectomy. Braz J Otolaryngol. 2011;77(4):510-5.

3. Albelda SM, Buck CA: Integrins and other cell adhesion molecules, FASEB J 4:2868, 1990.

4. Bevilacqua MP, Nelson RM: Selectins, J Clin Invest. 1993:91:379-85.

5. Lamas AM, Mulroney CR, Schleimer RP. Studies on the adhesive interaction between human eosinophils and cultured vascular endothelial cells. J Immunol. 1988;140(5):1500-5.

6. Copelli C, Manfuso A, d'Ecclesia A, Catanzaro S, Cassano L, Pederneschi N, et al. Endoscopic transnasal approach and intraoperative navigation for the treatment of isolated blowout fractures of the medial orbital wall. J Craniomaxillofac Surg. 2015;43(10):1974-8.
7. Svider PF, Baredes S, Eloy JA. Pitfalls in sinus surgery: an overview of complications. Otolaryngol Clin North Am. 2015;48(5):725-37.

8. Krings JG, Kallogjeri D, Wineland A, Nepple KG, Piccirillo JF, Getz AE. Complications of primary and revision functional endoscopic sinus surgery for chronic rhinosinusitis. Laryngoscope. 2014;124(4):838-45.

9. Scangas GA, Lehmann AE, Remenschneider AK, Su BM, Shrime $M G$, Metson R. The value of frontal sinusotomy for chronic rhinosinusitis with nasal polyps-A cost utility analysis. Laryngoscope. 2018;128(1):43-51.

10. Kutluhan A, Çetin H, Kale H, Kara Ö, Mişe Hï, Oğuzhan T, et al. Comparison of natural ostiodilatation and endoscopic sinus surgery in the same patient with chronic sinusitis. Braz J Otorhinolaryngol. 2018;1808(18):30763-8.

11. Chung YJ, An SY, Yeon JY, et al. Effect of a chitosan gel on hemostasis and prevention of adhesion after endoscopic sinus surgery. Clin Exp Otorhinolaryngology. 2016;9(2):143-9.

12. Ryu IY, Kwon C, Koh TK, Lee KH, Cho JS, Kim SW. Changes in Clinical Characteristics of the Patients Who Received Endoscopic Sinus Surgery according to 10 Year Temporal Difference: A Retrospective Observational Study. J Rhinol. 2016;23(2):81-4.

13. Noon E, Hopkins C. Review article: outcomes in endoscopic sinus surgery. BMC Ear Nose Throat Disord. 2016;16(2):9-15.

14. Clement Young, David S.Y. Sia, James T.P.D Hallinan and Vincent C, Imaging of the Paranasal Sinuses and Their Surgical Relevanc., Skull Base Imaging. 2018:16(5):21-38.

15. Shah RR, Thomas WW, Kuan EC, Kennedy DW. The lamina push test: an alternative to the globe push test for identifying the medial orbit during endoscopic sinus surgery. Int Forum Allergy Rhinol. 2018;8(9):1073-5.

16. Abuzeid WM, Peterson AZ, Rathor A, Xu JC, Velasquez N, Rashan AR, et al. Enhanced Irrigant Delivery to the Ethmoid Sinuses Directly Following Ethmoid Punch Sinusotomy. Ann Otol Rhinol Laryngol. 2017;126(12):804-12.

\section{AUTHORSHIP AND CONTRIBUTION DECLARATION}

\begin{tabular}{lc} 
AUTHORS & Contribution to The Paper \\
\hline Dr. Babar Rafiq Khan & Co-author, Layout of manuscript, Literature Review, \\
Associate Professor, ENT-I, Faisalabad Medical & Proof Reading \\
University, Allied Hospital, Faisalabad & Study designing, Data Collection / Analysis, \\
\hline Dr. Muhammad Saleem & Preparation of Results, \\
Assistant Professor, ENT, & \\
Aziz Fatima Medical \& Dental College, Faisalabad & Authentication of References \\
\hline Dr. Khizer Saleem & \\
House Officer, & Literature Review \\
Bahawalpur Victoria Hospital, Bahawalpur & \\
Dr. Jamil Ahmed & Literature Review \\
PHRC, Research Centre, \\
Faisalabad Medical University, Faisalabad
\end{tabular}

